\title{
Urologic Problems in Multiple Sclerosis
}

\author{
Srinivas Pentyala* ${ }^{1.2}$, Sadaf Jalali ${ }^{2}$, James Park ${ }^{2}$, Bilal Chughtai ${ }^{2}$, Praneet Korrapati ${ }^{1}$, Pooja \\ Mysore $^{1}$ and Sardar Ali Khan ${ }^{2}$
}

Department of ${ }^{1}$ Anesthesiology and ${ }^{2}$ Urology, Stony Brook Medical Center, Stony Brook, NY 11794, USA

\begin{abstract}
Multiple sclerosis (MS) is a devastating neurologic disease that attacks young adults and affects all aspects of their lives. Genitourinary dysfunction in MS has a significant impact on quality of life with more than $80 \%$ of those with MS reporting genitourinary symptoms. Voiding dysfunction is a prominent problem affecting the majority of MS sufferers. Less commonly recognized, but of no less importance, is sexual dysfunction which affects both men and women with MS. At least $75 \%$ of men and 50\% of women report sexual dysfunction and suspicions of under-reporting are strong. In this review, physiology of micturition, sexual functioning and disruptions in neural pathways occurring in MS that can result in the clinical syndromes of voiding dysfunction, sexual dysfunction and paraphilias will be discussed. Management of these disorders is also reviewed.
\end{abstract}

Keywords: Multiple sclerosis, urology, voiding dysfunction, sexual dysfunction, paraphilias, neurology

\section{INTRODUCTION}

Multiple sclerosis (MS) is a devastating autoimmune inflammatory neurologic disease that affects young adults with impairments in quality of life. The etiology of the disease continues to be under investigation, but both hereditary and environmental factors are believed to be at play. The disease is characterized by multiple demyelinating plaques with axonal sparing scattered throughout the central nervous system. All neurologically controlled functions are at risk in this debilitating and distressing disease. MS is not a rare disease affecting 1 in 1000 people in the United States and 2 in 1,000 northern Europeans, with a female to male ratio of 2:1. Generally the first manifestations appear in people aged 20-50 years old, but those as young as 5 and as old as 70 have also been diagnosed [1]. Multiple sclerosis has a significant impact on quality of life. Urologic dysfunction plays a central role with more than $80 \%$ of those with MS reporting genitourinary symptoms. Voiding dysfunction is a prominent problem affecting the majority of MS sufferers. After 10 years of disease, as much as $96 \%$ of the afflicted will have urodynamic findings of voiding dysfunction [2]. Less commonly recognized is sexual dysfunction which affects both men and women alike. At least $75 \%$ of men and $50 \%$ of women report sexual dysfunction, although the incidence may be greater due to under-reporting [3]. Both voiding and sexual function depend on intact neural pathways from brain to sacral cord. The disruption of these pathways results in various voiding and sexual syndromes that the astute clinician will easily diagnose.

*Address correspondence to this author at the Department of Anesthesiology, School of Medicine, Stony Brook Medical Center, Stony Brook, NY 11794-8480, USA; Tel: 631-444-2974; Fax: 631-4442908; Email: srinivas.pentyala@ stonybrook.edu; spentyala@notes.cc.sunysb.edu

\section{MULTIPLE SCLEROSIS}

Multiple sclerosis is an autoimmune inflammatory disorder of central nervous system white matter resulting in focal areas of oligodendrytic loss and reactive gliosis. Periventricular white matter and spinal tracts are predominantly involved, but every area of the central nervous system is at risk [2]. The focal demyelination disrupts saltatory conduction and slows or stops nerve transmission is affected areas. The pathological site of demyelination determines the major neurourological symptoms of the disease [4].

The pathogenesis of the disease is still largely not understood but theories with evidentiary support have been proposed [1]. The distinctive histopathological findings include perivenular lymphocytic infiltrates and macrophage insinuation in white matter [2]. The predominantly T-cell mediated autoimmune attack is primarily directed at the target myelin basic protein. The inciting event, resulting in a loss of tolerance is currently unknown, but several viruses are being investigated as potential etiologic factors. It is thought that because of molecular mimicry between self proteins and viral or bacterial proteins, tolerance to self antigens are lost, inciting autoimmunity. Another theory suggests direct infection with viruses as an ongoing event, causing a persistent inflammatory assault on oligodendrocytes. Studies in twins have shown that there is a hereditary component, but concordance is only $30 \%$ indicating that environmental factors are also at play [2].

\section{THE AUTONOMIC NERVOUS SYSTEM AND MICTURITION}

Micturition involves both the parasympathetic and sympathetic divisions of the autonomic nervous system as well as the somatic nervous system. Centers in the pons initiate and coordinate micturition by stimulating parasympathetic outflow and inhibiting somatic stimulation. 
Parasympathetic neurons in the intermediolateral column of S2-4 segments of the spinal cord are stimulated and send their preganglionic signals to postganglionic neurons in the pelvic plexus or in the detrusor muscle. Stimulation of these postganglionic fibers and subsequent release of acetylcholine triggers contraction of the detrusor muscle and relaxation of the bladder neck (internal smooth muscle sphincter) thus favoring bladder emptying. Simultaneous inhibition of somatic efferents originating in the sacral spinal cord brings about relaxation of the external striated sphincter favoring bladder emptying. Sympathetic fibers originating from neurons in the T10-L2 segments of the spinal cord have opposing effects and favor bladder filling. These preganglionic fibers are carried through hypogastric nerves and synapse on postganglionic fibers in pelvic ganglia. Subsequently postganglionic fibers release norepinephrine on beta receptors in the detrusor muscle and alpha receptors in the bladder neck promoting relaxation and contraction, respectively [5].

\section{MULTIPLE SCLEROSIS AND MICTURITION}

Lesions in the pons, lateral corticospinal and reticulospinal tracts and sacral cord neurons can have deleterious effects on voiding. The site of lesion can be correlated to the type of syndrome that develops [6]. Suprasacral lesions are commonly found in the cervical cord and can disrupt descending inhibitory signals resulting autonomous detrusor contractions producing irritative symptoms of frequency and urgency, and ultimately urge incontinence. Plaques in the reticulospinal tract interrupt fibers originating in the pons thus impairing synergistic contraction of the detrusor and relaxation of the external sphincter. Detrusor external sphincter dyssynergia (DESD) results and prevents efficient bladder emptying and possibly increased bladder pressures [2]. These lesions can also cause incomplete relaxation of the sphincter or paralysis and subsequent incontinence. Lesions in the pons commonly result in detrusor hyporeflexia since stimulatory signals are lost [6]. Sacral plaques are much less common being found in only $18 \%$ of MS patients at autopsy. A number of manifestations may result including hypocontraction or areflexia of the detrusor. Sensory afferents may also be disrupted causing a reduced awareness of bladder fullness and increased urinary retention [2]. Recurrent urinary tract infections (UTI) are common in MS probably due to incomplete bladder emptying. It was reported that parasympathetic dysfunction was more closely related to the progression of disability with MS, while sympathetic dysfunction was associated with the clinical activity [7].

\section{EVALUATION OF THE URINARY TRACT IN MS PATIENTS}

Incidence of high rates of overactive bladder symptoms in MS patients was observed with increased disease duration and physical disability. Despite an increasing awareness of overactive bladder symptoms and the need for evaluation and treatment, many MS patients remain underserved [8]. All patients have to undergo extensive history and physical examination focused on the urinary tract. Regardless of findings, all MS patients should be screened for UTI, urinary retention, and renal dysfunction with a urinalysis and urine culture, post void residual and BUN/creatinine and renal and bladder ultrasound. Patients with lower urinary tract symptoms including urgency, frequency, nocturia, urge incontinence and urinary retention should undergo baseline renal ultrasound and urodynamic studies because natural history of MS is undetermined. Upper urinary tract disease is uncommon in MS and can be delayed as long as bladder pressures are kept below $40 \mathrm{~cm}$ water as determined by urodynamic studies [9]. Risk factors for upper tract disease include filling pressures greater than $40 \mathrm{~cm}$ water, male DESD and presence of indwelling catheter [2].

The lower urinary tract should be evaluated with video urodynamics. Most frequently patients suffer from detrusor hyperreflexia with or without DESD [10]. However, this may occur together with or evolve into incomplete bladder emptying and urinary retention. Urodynamic evaluation can separate out these findings and allow the best and most complete treatment. These studies should be repeated at consistent intervals regardless of symptomatology since findings are likely to change, requiring a concomitant evolution in treatment [11]. Serial ultrasounds screening for hydronephrosis should be undertaken every 3-6 months on those patients with significant progress of lower urinary tract symptoms or rapid progression of MS as hydronephrosis is a silent disease associated with loss of renal function.. Post voiding residuals should be kept less than $100 \mathrm{ml}$ using clean intermittent catheterization (CIC) if necessary. Keeping the bladder collapsed to improve microcirculation of the detrusor muscle and eradicating bacterial growth in residual urine, can result in less episodes of urinary tract infection. Patient education regarding these parameters of urinary tract is essential in the long term management of those afflicted with MS.

\section{TREATMENT OF VOIDING DYSFUNCTION}

Voiding dysfunction in multiple sclerosis is usually amenable to medical treatment and rarely results in upper tract disease [12]. The mainstay of treatment for detrusor overactivity and urge incontinence are oral anticholinergic agents including oxybutynin and the more selective tolterodine. Those refractory to these medications may benefit from the anticholinergic effects of tricyclic antidepressants. Alternatively, a reduction in the production of urine may be helpful and can be achieved with nasal or oral desmopressin.

When oral treatment fails, intravesical treatments may be tried, but these methods are obviously less convenient and require dexterity adequate for instillation [12]. Agents effectively administered in this manner include anticholinergics, botulinum toxin and vanilloids, such as capsaicin. The former two act to decrease bladder contraction while the latter reduces sensation from the bladder [10]. Anecdotal evidence has led to increased interest in cannabis-based therapies for bladder symptoms, although exact pharmacological pathways are unknown [10].

Incomplete bladder emptying may be an isolated sign, in combination with detrusor over activity or as a result of its treatment. As such, treatments for incomplete emptying are often combined with the above treatments when there is evidence of urinary retention [2]. These treatments include clean intermittent catheterization, indwelling or suprapubic catheters as well as surgical urinary diversion for advanced 
disease. Novel treatments like remote controlled intraurethral In-Flow catheter [13], remote controlled intravesical device [14] and sacral nerve stimulation [15] are also considered in treating incontinence. Generally CIC is adequate when dexterity allows, but permanent catheterization or urinary diversion should be considered when advanced disease prevents CIC $[2,10]$.

Patients who are refractory to the above interventions have been found to respond to Botox intradetrusor injections. Despite slightly impaired detrusor contractility most patients still voided voluntarily without symptoms. Hence, Botox (100 units) may be a reasonable treatment option for overactive bladder symptoms in patients with multiple sclerosis who still void voluntarily [16]. Also percutaneous posterior tibial nerve stimulation treatment has been reported to be effective to suppress neurogenic detrusor overactivity in MS patients [17].

Other nonpharmacologic interventions include timed voiding, regulation of fluid intake, and reduced intake of bladder irritants such as aspartame and caffeine along with physiotherapy and pelvic exercises [18]. Surgical solutions represent a last resort approach since MS is usually unpredictably progressive, rendering surgery as an impermanent solution [2]. Treatment of UTIs should follow normal guidelines with determination of etiology of urinary tract infection and prophylactic regimes with recurrent infections with careful concomitant monitoring of renal and bladder ultrasound.

\section{NORMAL SEXUAL FUNCTION}

Sexual function in both men and women is a complex process mediated by neurologic, hormonal, vascular and psychological factors. Desire and libido are thought to be under cerebral control, separate from the physical sexual response. Both parasympathetic and sympathetic divisions of the autonomous nervous system have central roles in sexual functioning and follow paths similar to those described earlier in voiding. Male sexual cycle involves libido and arousal, penile erection, emission and ejaculation, orgasm, and detumescence. In men, the postganglionic fibers of both parasympathetic and sympathetic neurons are carried via the cavernosal nerves located posterolateral to the prostate to the corporal bodies. Sensory input from the penile and perineal areas is carried back to the dorsal root ganglia via branches of the pudendal nerve. Activation of parasympathetic fibers originating from S2-4 segments of the spinal cord stimulates erection by relaxing smooth muscle in the corporal bodies thereby promoting vascular engorgement and reduction of venous outflow. Sympathetic activation results in ejaculation and detumescence through contraction of the sinusoidal smooth muscle which decreases blood flow into the corpora allowing recommencement of venous outflow [5, 20]. Sexual functioning in women is very similar to that of men and involves excitement, plateau, orgasm and resolution. Arousal is also a neurovascular event marked by vascular engorgement of the labia, vaginal walls and clitoral body as well as vaginal lubrication. All of these actions are mediated through parasympathetic stimulation via pelvic nerves. Vaginal lubrication is further modulated through the actions of estrogen [19, 21].

\section{SEXUAL DYSFUNCTION IN MEN}

Sexual dysfunction in MS is rarely a presenting symptom but is very common in men greater than four years post diagnosis, affecting $70-91 \%$ of patients $[2,5]$. In men, sexual dysfunction manifests as decreased libido, decreased sensation, erectile dysfunction and disorders of orgasm [12]. Erectile dysfunction often presents at the same time as voiding dysfunction and lower motor dysfunction [22]. This is consistent with neural anatomy, since deteriorating spinal function will affect all functions mediated through suprasacral spinal tracts [2]. The defect may be partial initially, so that erectile dysfunction is variable. Morning and noctural erections may be preserved upon onset but usually wane with time [23]. Ejaculatory dysfunction more commonly manifests as difficulty in achieving ejaculation but occasionally premature ejaculation is also reported [12]. Anorgasmia and prolonged interval to orgasm are also frequently noted by male patients suffering from MS. Fatigue is a prominent complaint in MS and this will have effects on overall sexual functioning and ability to maintain sexual positioning.

Complicating the organic causes of sexual dysfunction in both male and female MS patients are the psychosocial aspects of chronic disease and medication side effects (Table 1). Depression and fatigue correlate well with sexual dysfunction in males [5]. Nocturnal penile tumescence studies have shown that there is a psychogenic component to erectile dysfunction in greater than $50 \%$ of those tested [2]. Self esteem and interpersonal issues are prevalent in patients with MS and contribute to decreased sexual satisfaction in relationships [5]. Evaluation of sexual dysfunction requires minimal testing and can be diagnosed on a clinical basis alone. Measure of serum testosterone will rule out hormonal etiologies and nocturnal penile tumescence can be undertaken if there is a suspicion of psychogenic erectile dysfunction [5, 24].

Table 1. Multifactorial Etiology of Male and Female Sexual Dysfunction in Patients with Multiple Sclerosis

\begin{tabular}{|ll|}
\hline - & Uro-genital Autonomic dysfunction \\
- & Motor and sensory disturbances \\
- & Embarrassment secondary to Urinary and Fecal Incontinence \\
- & MS induced Cranial nerves Neuropathy \\
- & MS induced Fatigue \\
- & MS induced Depression \\
- & Use of Antidepressants in Multiple Sclerosis \\
- & Pudendal Neuropathy in Multiple Sclerosis \\
- & Veno-occlusive dysfunction secondary to Endothelial \\
& Dysfunction of Neurovascular penile circulation
\end{tabular}

\section{SEXUAL DYSFUNCTION IN WOMEN}

Sexual dysfunction in females with MS is common, affecting $43-72 \%$ of patients [2, 12], reflecting neurological dysfunction, psychological factors, depression, side effects of medications and physical manifestations of the disease, such as fatigue and muscle weakness [25]. The majority of 
female MS sufferers would like to continue sexual activity but somatic and psychosocial problems resulting from MS make this difficult (Table 1). Female sexual dysfunction is manifested in a variety of disorders as classified by American Foundation for Urologic Disease [12] (Table 2).

Table 2. Female Sexual Function Disorders

\begin{tabular}{|c|c|}
\hline Sexual Desire Disorders & $\begin{array}{c}\text { Hypoactive Sexual Desire, Sexual } \\
\text { Aversion Disorder, }\end{array}$ \\
\hline \hline Sexual arousal disorders & $\begin{array}{c}\text { Decreased appetite, decreased } \\
\text { genital blood flow due to missed } \\
\text { arousal or dysphoric arousal }\end{array}$ \\
\hline Sexual pain disorders & Dyspareunia, vaginismus, other \\
\hline Orgasm disorders & $\begin{array}{c}\text { Increased interval to orgasm, } \\
\text { anorgasmia }\end{array}$ \\
\hline
\end{tabular}

Female patients with MS commonly report decreased libido, difficulty with arousal, decreased sensation, decreased lubrication, dyspareunia and anorgasmia [3, 12].Fatigue and recurrent UTIs also contribute to sexual dysfunction [2]. Difficult orgasm is the most common sexual complaint in female MS patients. This is attributed to impaired genital somatosensory afferents [26]. The psychosocial aspects of concurrent depression and difficulty with self esteem and interpersonal relationships are common in female MS patients and interfere with sexual satisfaction [3]. In up to $39 \%$ of women, sexual activity ceases or is unsatisfactory [27]. Rarely patients with MS can suffer from hypersexuality and paraphilias.

\section{PARAPHILIAS IN MS}

Paraphilias and hypersexuality are uncommon in MS but when these syndromes exist, the effects can be devastating. Men suffering form hypersexuality and paraphilia were eventually incarcerated $[1,28]$. Paraphilia was reported to be one of the presenting symptom of MS [28]. Sexual impulses are controlled in a complex and incompletely understood manner involving the subcortical structures such as the hypothalamus and septal area along with parts of the temporal and frontal lobes. Focal traumatic lesions in these areas have been shown to produce paraphilias and sexual disinhibition [28]. In patients with MS, case reports have identified men and women with the following manifestations: increased libido, increased and public masturbation, inappropriate sexual questioning and gesturing to strangers and exihibitionism, as well as fetishism, zoophilia, scopophilia, pedophilia and incest [1,28-30].

\section{TREATMENT OF SEXUAL DYSFUNCTION IN MEN}

The advent of phosphodiesterase inhibitors has revolutionized the treatment of erectile dysfunction and has proved effective in the male MS population [12]. The extent of improved sexual function is debated, as one double-blind placebo controlled study involving sildenafil citrate in MS patients showed no significant improvement whereas another study showed significant improvement in both erectile function and quality of life [31,32]. Injection therapy with prostaglandins has also shown excellent response rates in neurogenic erectile dysfunction but requires dexterity to administer. Over time patients with MS abandon this method even though patients are reporting a good response [12]. Vacuum devices have similar problems with dexterity but are good option if patients wish to avoid medications. A pilot trial found that over $50 \%$ of those who started using a vacuum device were still utilizing it after 21 months [33]. Semi-rigid penile implants over inflatable devices are a surgical alternative to medical treatment, as loss of dexterity may occur with MS patients and this will have an impact on the usage of inflatable devices. Counseling should also be offered since the etiology of sexual dysfunction is often multifactorial with psychosocial aspects being prominent [2].

\section{TREATMENT OF SEXUAL DYSFUNCTION IN WOMEN}

Treatment of sexual dysfunction in women has trailed that of men, but research is underway to test phosphodiesterase inhibitors and prostaglandins [12, 3436].Approved therapies to date focus on symptomatic improvements. Vaginal dryness due to decreased lubrication can be treated with oral or topical estrogens. Methyl testosterone can be used to increase libido [12]. Vibratory stimuli may be helpful in improving arousal [2]. Counseling is important to address psychosocial relationship concerns [2]. Symptomatic treatment of fatigue and variation in positioning may also be beneficial. UTIs should be treated promptly and prophylactic treatment initiated to avoid recurrent UTIs. Assessment and treatment of sexual problems in female MS patients should combine medical and psychosexual approaches and begin as soon as MS is diagnosed. Intervention can be done by recognizing sexual needs, and providing relevant education [25].

\section{TREATMENT OF HYPERSEXUALITY AND PARAPHILIAS}

Treatments for hypersexuality and paraphilias are scarce, but attempts include counseling, serotonin agonists, antiandrogens, steroids and gonadotropin-releasing hormone analogs. In the MS population these treatments have been unsuccessful but long term treatment was not achieved due to incarceration $[1,28,30]$.

\section{CONCLUSION}

Urological problems like voiding dysfunction, sexual dysfunction and also related paraphilias and hypersexuality associated with MS should be carefully evaluated before appropriate treatments are considered. Recognition and rigorous definition of urogenital dysfunction allows the clinician to offer the best treatment strategies discussed above, which provides symptomatic relief and improvements in the already fragile quality of life of MS patients.

\section{REFERENCES}

[1] Frohman EM, Racke MK, Raine CS. Multiple sclerosis--the plaque and its pathogenesis. N Engl J Med 2006; 354: 942-55.

[2] Litwiller SE, Frohman EM, Zimmern PE. Multiple sclerosis and the urologist. J Urol 1999; 161: 743-57.

[3] Jonsson A, [Disseminated sclerosis and sexuality]. Ugeskr Laeger 2003; 165: 2642-6.

[4] Araki I, Matsui M, Ozawa K, Nishimura M, Kuno S, Saida T. Relationship between urinary symptoms and disease-related parameters in multiple sclerosis. J Neurol 2002; 249: 1010-5.

[5] Fernandez O. Mechanisms and current treatments of urogenital dysfunction in multiple sclerosis. J Neurol 2002; 249: 1-8. 
[6] Araki I, Matsui M, Ozawa K, Takeda M, Kuno S. Relationship of bladder dysfunction to lesion site in multiple sclerosis. J Urol 2003; 169: 1384-7.

[7] Flachenecker P, Reiners K, Krauser M, Wolf A, Toyka KV. Autonomic dysfunction in multiple sclerosis is related to disease activity and progression of disability. Mult Scler 2001; 7: 327-34.

[8] Mahajan ST, Patel PB, Marrie RA. Under treatment of overactive bladder symptoms in patients with multiple sclerosis: an ancillary analysis of the NARCOMS Patient Registry. J Urol 2010; 183: $1432-7$.

[9] Lemack GE, Hawker K, Frohman E. Incidence of upper tract abnormalities in patients with neurovesical dysfunction secondary to multiple sclerosis: analysis of risk factors at initial urologic evaluation. Urology 2005; 65: 854-7.

[10] DasGupta R, Fowler CJ. Bladder, bowel and sexual dysfunction in multiple sclerosis: management strategies. Drugs 2003; 63: 153-66.

[11] Ciancio SJ, Mutchnik SE, Rivera VM, Boone TB. Urodynamic pattern changes in multiple sclerosis. Urology 2001; 57: 239-45.

[12] DasGupta R, Fowler CJ. Sexual and urological dysfunction in multiple sclerosis: better understanding and improved therapies. Curr Opin Neurol 2002; 15: 271-8.

[13] Madjar S, Sabo E, Halachmi S, et al. A remote controlled intraurethral insert for artificial voiding: a new concept for treating women with voiding dysfunction. J Urol 1999; 161: 895-8.

[14] Yachia D. New approach to the management of stress urinary incontinence in patients with treatment failure. J Endourol 2004; 18(3):211-4.

[15] Wexner SD, Coller JA, Devroede G, et al. Sacral nerve stimulation for fecal incontinence: results of a 120-patient prospective multicenter study. Ann Surg 2010; 251: 441-9.

[16] Mehnert U, Birzele J, Reuter K, Schurch B. The effect of botulinum toxin type a on overactive bladder symptoms in patients with multiple sclerosis: a pilot study. J Urol 2010; 184: 1011-6.

[17] Kabay S, Kabay SC, Yucel M, et al. The clinical and urodynamic results of a 3-month percutaneous posterior tibial nerve stimulation treatment in patients with multiple sclerosis-related neurogenic bladder dysfunction. Neurourol Urodyn 2009; 28: 964-8.

[18] Crayton HJ, Rossman HS. Managing the symptoms of multiple sclerosis: a multimodal approach. Clin Ther 2006; 28: 445-60.

[19] Traish AM, Kim NN, Munarriz R, Moreland R, Goldstein I. Biochemical and physiological mechanisms of female genital sexual arousal. Arch Sex Behav 2002; 31: 393-400.

[20] Masters WH, Johnson VE. The sexual response of the human male. I. Gross anatomic considerations. West J Surg Obstet Gynecol 1963; 71: 85-95.
[21] Masters WH, Johnson VE. The human female: anatomy of sexual response. Minn Med 1960; 43: 31-6.

[22] McDougall AJ, McLeod JG. Autonomic nervous system function in multiple sclerosis. J Neurol Sci 2003; 215: 79-85.

[23] Rees PM, Fowler CJ, Maas CP. Sexual function in men and women with neurological disorders. Lancet 2007; 369: 512-25.

[24] Haensch CA, Jorg J. Autonomic dysfunction in multiple sclerosis. J Neurol 2006; 253 Suppl 1: I3-9.

[25] Bronner G, Elran E, Golomb J, Korczyn AD. Female sexuality in multiple sclerosis: the multidimensional nature of the problem and the intervention. Acta Neurol Scand 2010; 121: 289-301.

[26] Yang CC, Bowen JR, Kraft GH, Uchio EM, Kromm BG. Cortical evoked potentials of the dorsal nerve of the clitoris and female sexual dysfunction in multiple sclerosis. J Urol 2000; 164: 2010-3.

[27] Salonia A, Munarriz RM, Naspro R, et al. Women's sexual dysfunction: a pathophysiological review. BJU Int 2004; 93: 115664.

[28] Huws R, Shubsachs AP, Taylor PJ. Hypersexuality, fetishism and multiple sclerosis. Br J Psychiatry 1991; 158: 280-1.

[29] Gondim FA, Thomas FP. Episodic hyperlibidinism in multiple sclerosis. Mult Scler (2001); 7: 67-70.

[30] Yang CC, Severson B, Bowen JD. Hypersexual sensations and behavior in a multiple sclerosis exacerbation: a case report. Int $\mathrm{J}$ Impot Res 2004; 16: 382-4.

[31] Fowler CJ, Miller JR, Sharief MK, Hussain IF, Stecher VJ, Sweeney M. A double blind, randomised study of sildenafil citrate for erectile dysfunction in men with multiple sclerosis. J Neurol Neurosurg Psychiatry 2005; 76: 700-5.

[32] Safarinejad MR. Evaluation of the safety and efficacy of sildenafil citrate for erectile dysfunction in men with multiple sclerosis: a double-blind, placebo controlled, randomized study. J Urol 2009; 181: 252-8.

[33] Heller L, Keren O, Aloni R, Davidoff G. An open trial of vacuum penile tumescence: constriction therapy for neurological impotence. Paraplegia 1992; 30: 550-3.

[34] Chivers ML, Rosen RC. Phosphodiesterase type 5 inhibitors and female sexual response: faulty protocols or paradigms? J Sex Med 2010; 7: 858-72.

[35] Brotto LA, Bitzer J, Laan E, Leiblum S, Luria M. Women's sexual desire and arousal disorders. J Sex Med 2010; 7: 586-614.

[36] Bitzer J, Alder J. Female sexual dysfunction. Ther Umsch 2010: 67: 105-16. 